\title{
Comparative Effectiveness of Ustekinumab Versus Adalimumab in Induction of Clinical Response and Remission in Crohn's Disease: Experience of a Real-World Cohort at a Tertiary Care Inflammatory Bowel Disease Referral Center
}

\author{
Zunirah Ahmed $^{\mathrm{a}}$, Krishna Venkata ${ }^{\mathrm{b}}$, Nan Zhang ${ }^{\mathrm{c}}$, Talha A. Malik ${ }^{\mathrm{d}, \mathrm{e}}$
}

\begin{abstract}
Background: There is paucity of head-to-head studies comparing the effectiveness of ustekinumab (UST) and adalimumab (ADA) in Crohn's disease (CD). Here we provide a real-world comparison of these two agents.

Methods: We conducted an ambidirectional cohort study. Each patient included had moderate to severe active $\mathrm{CD}$. Clinical response and remission were assessed between 4 and 16 weeks after induction.

Results: Of a total of 163 patients, 97 were induced with ADA and 66 were induced with UST. Logistic regression model analysis adjusted based on effect size showed that ADA when compared to UST induced clinical response ( $73.2 \%$ vs. $50 \%$ (odds ratio (OR): 2.40 ; 95\% confidence interval $(\mathrm{CI}): 1.14-5.07 ; \mathrm{P}=0.02))$ and remission $(44.3 \%$ vs. $27.7 \%$ (OR: $2.35 ; 95 \%$ CI: $1.07-5.16$; $\mathrm{P}=0.034)$ in a statistically significantly higher proportion of patients. Among tumor necrosis factor (TNF)-naive patients, when comparing ADA vs. UST, ADA was superior in inducing clinical response $(69 / 89$ (77.5\%) vs. 4/10 (40\%) (OR: 4.26; 95\% CI: 1.08 - 16.84; P = 0.04)), but not remission $(41 / 89$ (46\%) vs. $3 / 9$ (33\%) (OR: $1.64 ; 95 \% \mathrm{CI}$ : 0.39 - 6.97; $\mathrm{P}=0.503)$ ). Among TNF-experienced patients, ADA was numerically inferior in inducing clinical response $(2 / 8(25 \%)$ vs. 29/56 (52\%) (OR: 0.38; 95\% CI: $0.07-1.94 ; \mathrm{P}=0.24)$ ) and remission $(2 / 8(25 \%)$ vs. $15 / 56(27 \%)$ (OR: 1.22 ; 95\% CI: 0.22 $6.81 ; \mathrm{P}=0.82)$ ), but neither of these differences were statistically significant.
\end{abstract}

Manuscript submitted May 24, 2019, accepted June 11, 2019

aDepartment of Medicine, University of Alabama at Birmingham, Montgomery, AL 36116, USA

${ }^{b}$ Hospitalist Medicine, Baptist South, Montgomery, AL 36116, USA 'Department of Biostatistics, Mayo Clinic, Scottsdale, AZ 85260, USA ${ }^{\mathrm{d}}$ Department of Medicine-Gastroenterology, Mayo Clinic, Scottsdale, AZ 85260, USA

${ }^{\text {e}}$ Corresponding Author: Talha A. Malik, Department of Medicine-Gastroenterology, Mayo Clinic, Scottsdale, AZ 85260, USA. Email: malik.talha@ mayo.edu

doi: https://doi.org/10.14740/gr1194
Conclusions: In a real-world setting, the rate of clinical response and remission was higher among patients with $\mathrm{CD}$ who received ADA compared to UST. Of note, however, despite the small sample sizes of TNF-experienced patients who received ADA and TNF-naive patients who received UST, the higher effectiveness of ADA in inducing clinical response and indeed remission among patients with $\mathrm{CD}$ with active disease appears to primarily be driven by those who are TNF-naive. Among TNF-experienced patients, UST may be superior in inducing clinical response and equally effective in inducing clinical remission when compared to ADA. Based on this study, one may infer that among TNF-experienced patients with CD with active disease, one could consider switching to an agent such as UST instead of a second approved TNF blocker. However, larger studies comparing the two agents are required.

Keywords: Crohn's disease; Adalimumab; Ustekinumab; TNF-experienced; TNF-naive; Clinical response; Clinical remission; Efficacy

\section{Introduction}

Crohn's disease (CD), characterized by transmural inflammation and skip lesion, is a heterogenous immune-mediated predominantly intestinal inflammatory condition that can affect any part of the gastrointestinal tract from mouth to the anus $[1,2]$. The annual incidence of $\mathrm{CD}$ ranges from 3 to 20 cases per 100,000 [3] with median age of onset of 30 years with the first peak at $20-30$ years and another peak at 50 years [2]. The natural course of the disease is relapsing and remitting in nature with a myriad of presentations ranging from inflammatory to stricturing and penetrating phenotypes [1]. Presenting symptoms are variable depending on disease severity. At least approximately one-third of patients develop perianal disease [4]. Extra-intestinal manifestations of CD occur in up to $25 \%$ of affected patients. These include erythema nodosum, pyoderma gangrenosum, iritis/uveitis and ankylosing spondylitis [5]. The pathogenesis of $\mathrm{CD}$ is complex, involving genetic predisposition, environmental factors, intestinal immune mechanisms and microbial flora [6]. T cells are the central effector cells and their soluble mediators (cytokines) are the key modulators in the dis- 
ease process [7]. Overproduction of several pro-inflammatory cytokines marks the disease process [8]. Therefore, the goal of treatment is rapid induction of steroid-free remission and prevention of long-term disease complications by inhibiting these mechanisms of inflammation. Treatment of CD depends on disease severity and phenotype. Conventional treatment modalities included steroids, mesalamine agents, thiopurine analogues and methotrexate until biological agents, specifically tumor necrosis factor (TNF) blockers such as infliximab and adalimum$\mathrm{ab}$ (ADA) took center stage for $\mathrm{CD}$ patients with moderate to severe disease [9]. Since the advent of TNF blockers, other biological agents have also been approved for use in CD. Most recently, ustekinumab (UST), a humanized monoclonal antibody against interleukin (IL)-12 and IL-23, has been approved for use in moderate to severe CD. Based on its encouraging safety profile, UST provides a promising treatment choice in patients with moderate to severe CD [10]. In this study, we sought to compare clinical response and remission to induction among patients with moderate to severe $\mathrm{CD}$ who received these agents in hopes of providing clinicians with a better understanding of their respective effectiveness in the real world.

\section{Materials and Methods}

\section{Study design, patient population and selection criteria}

We conducted an ambidirectional cohort study comparing effectiveness of induction with UST vs. ADA in patients with moderate to severe $\mathrm{CD}$ at University of Alabama at Birmingham (UAB), a tertiary care inflammatory bowel disease (IBD) referral center. The study population included adult $\mathrm{CD}$ patients seen at the UAB IBD center from 2014 to 2017. Inclusion criteria included patients aged 18 years or older at first observation (initial clinic visit) with clinically measured moderate to severe active CD who were induced with either UST or ADA within 12 weeks of the first clinic visit and followed until assessment of response or remission at a subsequent encounter falling between 4 and 16 weeks from the date of induction. Exclusion criteria included patients with poor or incomplete electronic medical record (EMR) documentation, those who were diagnosed with colorectal or another cancer, developed any severe infection or reaction, underwent any CD-related surgery, had a CD-related hospital admission, and women who were noted to be pregnant during the period of induction.

\section{Data collection and variable definitions}

Data were collected through retrospective as well as prospective review of EMR. Data collected at the time of the first observation in our tertiary referral center included age, race, gender, duration of disease, location and behavior of CD, nicotine use, proton pump inhibitor (PPI) use, vitamin D level, bone mineral density, presence of metabolic syndrome and its components, and biologic (vedolizumab/TNF blocker) experience. Data collected from the full period of observation included time from first clinical contact to induction as well as time from induction to assessment of clinical response. Data on additional $\mathrm{CD}$ therapy during induction (i.e. steroids, thiopurine analogue and methotrexate) was also collected. The exposure of interest comprised CD patients with moderately to severely active disease (Harvey Bradshaw index or HBI score of 8 or higher) who were induced with UST, and the comparison group included those with HBI score of 8 or higher induced with ADA. "Primary outcome" was clinical response (defined as fall in HBI score of at least 2 with a maximum score of 7 at assessment of response) measured between 4 and 16 weeks after induction. The secondary outcome was clinical remission (defined as those with clinical response and an overall HBI score of less than 4 at follow-up assessment) measured between 4 and 16 weeks after induction.

Nicotine use was defined as documented ongoing use at initial visit. PPI use was defined based on medication documentation in EMR at first visit. Steroid use was defined as exposure post- induction to rectal, topical, or oral corticosteroids for at least 4 weeks. Thiopurine use was defined as use of azathioprine or 6-mercaptopurine for at least 4 weeks post-induction.

Methotrexate use was defined as use of methotrexate for at least 4 weeks post-induction. Montreal classification was used to define location and behavior of $\mathrm{CD}$.

\section{Statistical analysis}

We conducted descriptive analysis for covariates by exposure groups (UST vs. ADA). Two sample $t$-test or Wilcoxon rank sum test was used to compare continuous variables and Chisquare test was used to compare categorical variables. Unadjusted and adjusted logistic regression models were used to estimate odds ratios (ORs) and 95\% confidence intervals $(95 \%$ CIs) for clinical response and remission. Potential confounders for inclusion into adjusted logistic regression models were selected based on their clinical relevance. For the overall population model, age, gender, race, duration of disease and vedolizumab experience were adjusted in the model. For the subgroup analysis, age and gender were adjusted as per the rule of thumb for logistic regression - 10 events (or non-events) per predictor. All statistical analyses were conducted using SAS 9.4. This study was conducted in compliance with the ethical standards of the responsible institution on human subjects as well as with the Helsinki Declaration. The current study was approved by UAB's Office of Institutional Review Board and informed consent was waived.

\section{Results}

Of 267 patients who received either UST or ADA during initial review of EMR, 100 and 167 were induced with UST and ADA, respectively. After eligibility criteria were applied, the final sample size included 66 patients induced with UST and 97 patients who received ADA. Of the 66 patients who were induced with UST, almost all $(\mathrm{n}=56)$ were TNF-experienced with only 10 being TNF-naive. Conversely, of the 97 patients who were induced with ADA, almost all $(n=89)$ were TNF- 


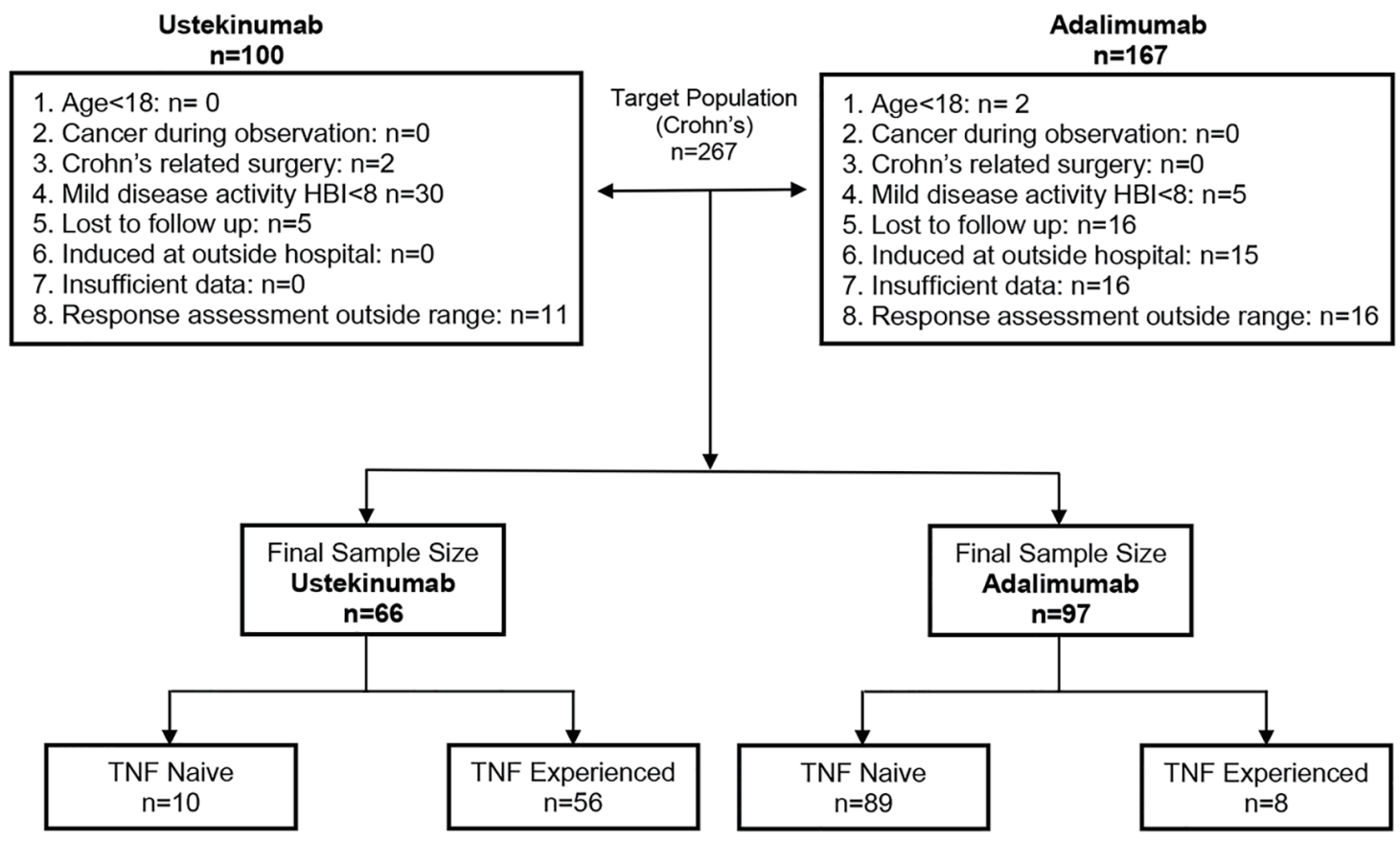

Figure 1. Flow chart showing the criteria for included patients.

naive with only eight $(8.2 \%)$ being TNF-experienced. Figure 1 shows the selection criteria of included patients.

Baseline characteristics of CD patients included in the final sample for the study by UST vs. ADA induction are shown in Table 1.

Clinical response was seen in 33/66 (50.0\%) patients induced with UST vs. $71 / 97$ (73.2\%) patients induced with ADA. The difference in response achieved statistical significance (odds ratio (OR): 2.40; 95\% confidence interval (CI): 1.14 - 5.07; $\mathrm{P}=0.02$ ), after adjusting for age, gender, race, nicotine use and vedolizumab experience (Table 2).

Clinical remission was achieved in $18 / 65$ patients induced with UST (27.7\%) vs. 43/97 patients (44.3\%) induced with ADA with likelihood of clinical remission being statistically significantly higher among CD patients induced with ADA compared to those who received UST (OR: $2.35 ; 95 \%$ CI: 1.07 - 5.16; $\mathrm{P}=0.0337$ ) (Table 3).

Among TNF-naive patients, when comparing ADA vs. UST, ADA was superior in inducing clinical response in 69/89 $(77.5 \%)$ vs. $4 / 10$ (40\%) patients (OR: 4.26; 95\% CI: 1.08 $16.84 ; \mathrm{P}=0.04)$ (Table 4) but not remission in $41 / 89(46 \%)$ vs. 3/9 (33\%) patients (OR: 1.64; 95\% CI: 0.39 - 6.97; $\mathrm{P}=$ 0.503) (Table 5). Among TNF-experienced patients, ADA was numerically inferior in inducing clinical response in $2 / 8(25 \%)$ vs. 29/56 (52\%) patients (OR: 0.38; 95\% CI: $0.07-1.94 ; \mathrm{P}=$ $0.24)$ (Table 6) and remission in $2 / 8(25 \%)$ vs. $15 / 56(27 \%)$ patients (OR: 1.22; 95\% CI: $0.22-6.81 ; \mathrm{P}=0.82$ ) (Table 7).

\section{Discussion}

The development of anti-TNF agents was a breakthrough in treatment for the multifactorial immune-mediated inflammatory disease that is CD. However, despite significant clinical response and remission rates to anti-TNF agents, around 30$40 \%$ of patients are primary non-responders and $20-30 \%$ of patients per year experience secondary loss of response [11]. For this significant subset of the patient population, newer biological agents have emerged as important treatment modalities [12]. UST is a fully human monoclonal antibody that blocks the IL-12/IL-23 p40 subunit and prevents its interaction with the common receptor IL-12R $\beta 1$, leading to reduced cytokine production and subsequent inflammatory process which characterizes the pathogenesis of CD [13].

Our study compared the effectiveness of UST in inducing clinical response and remission to anti-TNF agent ADA. Most patients who received UST were TNF-experienced, while most patients who received ADA were TNF-naive. Overall the likelihood of clinical response and remission to induction with UST appears to be much lower than the likelihood of response and remission to ADA. Of note however, UST appeared to be superior in inducing clinical response and remission when compared to ADA in TNF-experienced patients, while ADA was more effective in TNF-naive patients.

The landscape for treatment of inflammatory bowel diseases, particularly $\mathrm{CD}$, is evolving.

UST was initially approved for treatment of psoriasis [14]. The landmark study leading to its approval in moderate to severe CD was the UNIT1 trial in 2016 which evaluated the response in TNF-naive and TNF-experienced individuals where greatest clinical efficacy was noted in TNF-naive individuals [15]. Beyond the UNIT1 trial, the efficacy of UST has also been demonstrated in similar clinical trials for induction and maintenance of clinical response and remission in $\mathrm{CD}$ patients, 
Table 1. Baseline Characteristics of Included Patients

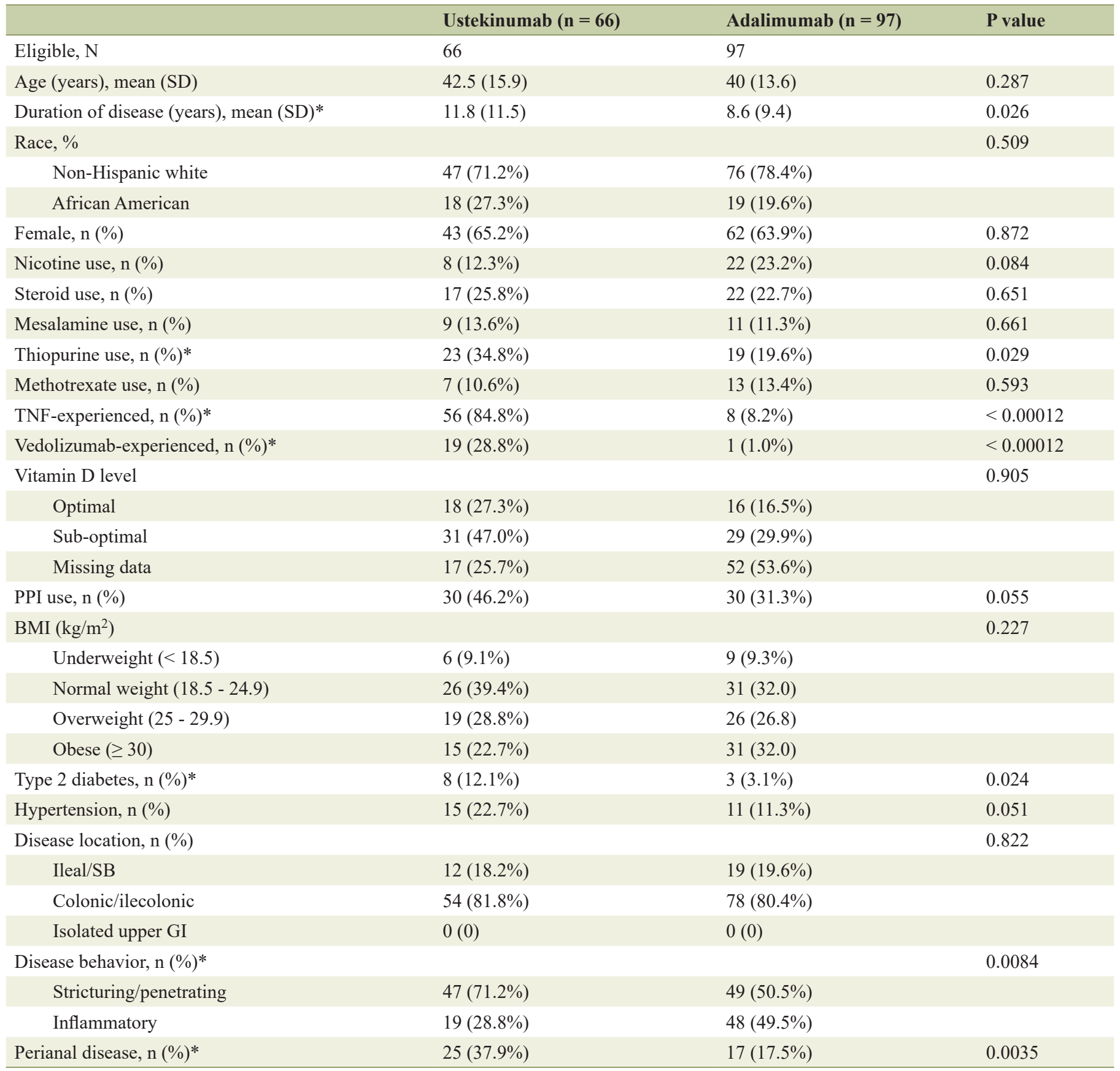

SD: standard deviation; TNF: tumor necrosis factor; BMI: body mass index; PPI: proton pump inhibitor; SB: small bowel; GI: gastrointestinal.

independent of their previous exposure to anti-TNF agents, for example in UNITI $2[10,16]$.

Multiple real-life cohorts have evaluated the effectiveness of UST in moderate to severe CD patients, either resistant to anti-TNF and conventional therapies or with intolerant side effects to TNF inhibitors. In these studies, UST was successful in improving clinical, laboratory, radiologic and endoscopic markers of disease activity in patients with severe, refractory CD unresponsive to TNF inhibitors [13, 17-22]. While it is known that TNF blocker exposure appears to reduce the likelihood of response among patients receiving UST or ADA, the difference in incidence of response and remission between the two groups in this study was despite that, still especially stark $[23,24]$.

This study had several limitations. The most important limitation was the small sample size which may impact generalizability. Another limitation was the observational and mostly retrospective nature of this study. Furthermore, several 
Table 2. Proportions, Crude and Adjusted Odds Ratios for Clinical Response in All Patients

\begin{tabular}{lllll}
\hline Group & Total, N & Clinical response, N (\%) & Unadjusted OR (95\% CI), P-value & Adjusted* OR (95\% CI), P-value \\
\hline Adalimumab & 97 & $71(73.2 \%)$ & $2.73(1.41-5.28), 0.0028$ & $2.40(1.14-5.07), 0.0213$ \\
Ustekinumab & 66 & $33(50.0 \%)$ & & \\
\hline
\end{tabular}

*Adjusted for age, gender, race, duration of disease and vedolizumab experience.

Table 3. Proportions, Crude and Adjusted ORs for Clinical Remission in All Patients

\begin{tabular}{lllll}
\hline Group & Total, N & Clinical remission, N (\%) & Unadjusted OR (95\% CI), P-value & Adjusted* OR (95\% CI), P-value \\
\hline Adalimumab & 97 & $43(44.3 \%)$ & $2.08(1.06-4.08), 0.0336$ & $2.35(1.07-5.16), 0.0337$ \\
Ustekinumab & 65 & $18(27.7 \%)$ & & \\
\hline
\end{tabular}

*Adjusted for age, gender, race, duration of disease and vedolizumab experience. OR: odds ratio; TNF: tumor necrosis factor; Cl: confidence interval.

Table 4. Proportions, Crude and Adjusted ORs for Clinical Response in TNF Naive Patients

\begin{tabular}{lllll}
\hline Group & Total, N & Clinical response, N (\%) & Unadjusted OR (95\% CI), P-value & Adjusted* OR (95\% CI), P-value \\
\hline Adalimumab & 89 & $69(77.5 \%)$ & $5.17(1.33-20.15), 0.0178$ & $4.26(1.08-16.84), 0.0390$ \\
Ustekinumab & 10 & $4(40.0 \%)$ & & \\
\hline
\end{tabular}

*Adjusted for age and gender. OR: odds ratio; TNF: tumor necrosis factor; Cl: confidence interval.

factors had to be adjusted because of their effect size. In future studies, the relationship between these variables and the treatment agents can be studied in more detail. Additionally, this study examined only clinical response and remission based on physician assessment and the HBI. Data on baseline biochemical, radiologic, or endoscopic parameters were not collected nor were these additional parameters examined in assessment of response.

Nonetheless, this study is a real-world reflection of the utility of UST vs. ADA in moderate to severe CD at a tertiary care IBD referral center as it stands today.

\section{Conclusion}

UST has emerged as a novel therapy for treatment of moderate to severe CD refractory to conventional treatments. It has been studied after the failure of TNF inhibitors and conventional therapies [25]. However, its role as first-line biological agent in achieving better clinical outcomes when directly compared

Table 5. Proportions, Crude and Adjusted ORs for Clinical Remission in TNF Naive Patients

\begin{tabular}{lllll}
\hline Group & Total, $\mathbf{N}$ & Clinical remission, N (\%) & Unadjusted OR (95\% CI), P-value & Adjusted* OR (95\% CI), P-value \\
\hline Adalimumab & 89 & $41(46.1 \%)$ & $1.71(0.40-7.26), 0.4684$ & $1.64(0.39-6.97), 0.5033$ \\
Ustekinumab & 9 & $3(33.3 \%)$ & & \\
\hline
\end{tabular}

*Adjusted for age and gender. OR: odds ratio; TNF: tumor necrosis factor; Cl: confidence interval.

Table 6. Proportions, Crude and Adjusted ORs for Clinical Response in TNF Experienced Patients

\begin{tabular}{lllll}
\hline Group & Total, $\mathbf{N}$ & Clinical response, N (\%) & Unadjusted OR (95\% CI), P-value & Adjusted* OR (95\% CI), P-value \\
\hline Adalimumab & 8 & $2(25.0 \%)$ & $0.31(0.06-1.67), 0.1732$ & $0.38(0.07-1.94), 0.2441$ \\
Ustekinumab & 56 & $29(51.8 \%)$ & & \\
\hline
\end{tabular}

*Adjusted for age and gender. OR: odds ratio; TNF: tumor necrosis factor; Cl: confidence interval.

Table 7. Proportions, Crude and Adjusted ORs for Clinical Remission in TNF Experienced Patients

\begin{tabular}{lllll}
\hline Group & Total, $\mathbf{N}$ & Clinical remission, N (\%) & Unadjusted OR (95\% CI), P-value & Adjusted* OR (95\% CI), P-value \\
\hline Adalimumab & 8 & $2(25.0 \%)$ & $0.91(0.17-5.02), 0.9148$ & $1.22(0.22-6.81), 0.8201$ \\
\hline Ustekinumab & 56 & $15(26.8 \%)$ & & \\
\hline
\end{tabular}

*Adjusted for age and gender. OR: odds ratio; TNF: tumor necrosis factor; Cl: confidence interval. 
to TNF blockers is unclear. Moreover, its long-term position as the second-line agent of choice after TNF failure also needs further evaluation. This is primarily driven by the lack of clinical data comparing UST to other agents especially among biological agent-naive specifically TNF blocker-naive patients. There is a pressing need for large multi-center studies assessing the clinical utility, efficacy and safety of UST in the management of CD when compared to other therapeutic options.

References

\section{Acknowledgments}

We would like to acknowledge the contribution of all the authors involved in the study.

\section{Financial Disclosure}

This study was not funded by any grant and the authors do not have any financial disclosures to make.

\section{Conflict of Interest}

The authors have declared no competing interests.

\section{Informed Consent}

The current study was approved by UAB's Office of Institutional Review Board and informed consent was waived.

\section{Author Contributions}

ZA: data collection and manuscript writing; $\mathrm{KV}$ : study design, data collection and manuscript writing; NZ: data analysis and reviewed the manuscript for important intellectual content; TM: study design and reviewed the manuscript for important intellectual content.

1. Ranasinghe IR, Hsu R. Crohn disease. In: StatPearls. Treasure Island (FL). 2019.

2. Feuerstein JD, Cheifetz AS. Crohn disease: epidemiology, diagnosis, and management. Mayo Clin Proc. 2017;92(7):1088-1103.

3. Molodecky NA, Soon IS, Rabi DM, Ghali WA, Ferris M, Chernoff G, Benchimol EI, et al. Increasing incidence and prevalence of the inflammatory bowel diseases with time, based on systematic review. Gastroenterology. 2012;142(1):46-54 e42; quiz e30.

4. Wilkins T, Jarvis K, Patel J. Diagnosis and management of Crohn's disease. Am Fam Physician. 2011;84(12):13651375.

5. Ephgrave K. Extra-intestinal manifestations of Crohn's disease. Surg Clin North Am. 2007;87(3):673-680.

6. Sartor RB. Mechanisms of disease: pathogenesis of Crohn's disease and ulcerative colitis. Nat Clin Pract Gas- troenterol Hepatol. 2006;3(7):390-407.

7. Danese S, Fiocchi C. Etiopathogenesis of inflammatory bowel diseases. World J Gastroenterol. 2006;12(30):48074812.

8. Cheifetz AS. Management of active Crohn disease. JAMA. 2013;309(20):2150-2158.

9. Colombel JF, Sandborn WJ, Reinisch W, Mantzaris GJ, Kornbluth A, Rachmilewitz D, Lichtiger S, et al. Infliximab, azathioprine, or combination therapy for Crohn's disease. N Engl J Med. 2010;362(15):1383-1395.

10. Sandborn WJ, Gasink C, Gao LL, Blank MA, Johanns J, Guzzo C, Sands BE, et al. Ustekinumab induction and maintenance therapy in refractory Crohn's disease. N Engl J Med. 2012;367(16):1519-1528.

11. Qiu Y, Chen BL, Mao R, Zhang SH, He Y, Zeng ZR, Ben-Horin $\mathrm{S}$, et al. Systematic review with meta-analysis: loss of response and requirement of anti-TNFalpha dose intensification in Crohn's disease. J Gastroenterol. 2017;52(5):535-554.

12. Seo GS, Lee SH. [Emerging therapies: what are promising in the near future?]. The Korean Journal of Gastroenterology. Taehan Sohwagi Hakhoe Chi. 2018;71:81-88.

13. Kotze PG, Ma C, Almutairdi A, Panaccione R. Clinical utility of ustekinumab in Crohn's disease. J Inflamm Res. 2018;11:35-47.

14. Gottlieb A, Narang K. Ustekinumab in the treatment of psoriatic arthritis: latest findings and clinical potential. Ther Adv Musculoskelet Dis. 2013;5(5):277-285.

15. Feagan BG, Sandborn WJ, Gasink C, Jacobstein D, Lang Y, Friedman JR, Blank MA, et al. Ustekinumab as induction and maintenance therapy for Crohn's disease. N Engl J Med. 2016;375(20):1946-1960.

16. Sandborn WJ, Feagan BG, Fedorak RN, Scherl E, Fleisher MR, Katz S, Johanns J, et al. A randomized trial of Ustekinumab, a human interleukin-12/23 monoclonal antibody, in patients with moderate-to-severe Crohn's disease. Gastroenterology. 2008;135(4):1130-1141.

17. Battat R, Kopylov U, Bessissow T, Bitton A, Cohen A, Jain A, Martel M, et al. Association Between Ustekinumab Trough Concentrations and Clinical, Biomarker, and Endoscopic Outcomes in Patients With Crohn's Disease. Clin Gastroenterol Hepatol. 2017;15(9):1427-1434 e1422.

18. Kopylov U, Afif W, Cohen A, Bitton A, Wild G, Bessissow T, Wyse J, et al. Subcutaneous ustekinumab for the treatment of anti-TNF resistant Crohn's disease - the McGill experience. J Crohns Colitis. 2014;8(11):15161522.

19. Ma C, Fedorak RN, Kaplan GG, Dieleman LA, Devlin SM, Stern N, Kroeker KI, et al. Clinical, endoscopic and radiographic outcomes with ustekinumab in medically-refractory Crohn's disease: real world experience from a multicentre cohort. Aliment Pharmacol Ther. 2017;45(9):1232-1243.

20. Greenup AJ, Rosenfeld G, Bressler B. Ustekinumab use in Crohn's disease: a Canadian tertiary care centre experience. Scand J Gastroenterol. 2017;52(12):1354-1359.

21. Wils P, Bouhnik Y, Michetti P, Flourie B, Brixi H, Bourrier A, Allez M, et al. Subcutaneous ustekinumab provides 
clinical benefit for two-thirds of patients with Crohn's disease refractory to anti-tumor necrosis factor agents. Clin Gastroenterol Hepatol. 2016;14(2):242-250 e241-242.

22. Ma C, Fedorak RN, Kaplan GG, Dieleman LA, Devlin SM, Stern N, Kroeker KI, et al. Long-term Maintenance of Clinical, Endoscopic, and Radiographic Response to Ustekinumab in Moderate-to-Severe Crohn's Disease: Real-world Experience from a Multicenter Cohort Study. Inflamm Bowel Dis. 2017;23(5):833-839.

23. Gendelman O, Weitzman D, Rosenberg V, Shalev V, Chodick G, Amital H. Characterization of adherence and persistence profile in a real-life population of patients treated with adalimumab. Br J Clin Pharmacol. 2018;84(4):786-795.

24. Knyazev OV, Kagramanova AV, Ruchkina IN, Fadeeva NA, Lishchinskaya AA, Boldyreva ON, Zhulina EY, et al. [Efficacy of adalimumab for Crohn's disease in real clinical practice]. Ter Arkh. 2017;89(2):20-27.

25. Danese S, Bonovas S, Peyrin-Biroulet L. Positioning ustekinumab in Crohn's disease: from clinical evidence to clinical practice. J Crohns Colitis. 2017;11(10):12581266. 